\title{
Cell Gap Effect on the Dynamics of Liquid Crystal Phase Modulators
}

\section{Haiying Wang \\ Xiangyi Nie \\ Thomas X. Wu}

School of Electrical Engineering and Computer Science, University

of Central Florida, Orlando, Florida, USA

\section{Shin-Tson Wu}

College of Optics and Photonics, University of Central Florida,

Orlando, Florida, USA

This paper provides a complete physical picture of optical response time as a function of cell gap (d) in a wide voltage regime. Normally both rise and decay times are known to be proportional to $d^{2}$ in the small angle approximation $\left(V_{\text {th }}<V<\sqrt{2} V_{t h}\right)$, and in the high voltage regime where $V_{\pi}<V<V_{i}$ the optical decay time is independent of $d$. In this paper, we found that between these two extremes there is a region where the response time is linearly proportional to $d$. Our analytical derivation is confirmed by experimental results.

Keywords: cell gap effect; optical response time; small angle approximation; spatial light modulator; transient nematic effect

\section{INTRODUCTION}

Liquid crystal (LC) spatial light modulator (SLM)has been used as a phase-only modulation for laser beam steering [1], tunable-focus lens [2-6], and other photonic devices [7-8]. To obtain a large phase shift while keeping operating voltage below $10 \mathrm{~V}_{\text {rms }}$, homogeneous cell (also called parallel-aligned cell) is a favorable choice. Response time of a homogeneous cell is a critical issue. To achieve a fast response time, low rotational viscosity $\left(\gamma_{1}\right)$ LC mixtures are preferred [9-11]. Another straightforward approach is to use a thin cell gap filled with a high

Address correspondence to Shin-Tson Wu, College of Optics and Photonics, University of Central Florida, Orlando, FL 32816, USA. E-mail: swu@mail.ucf.edu 


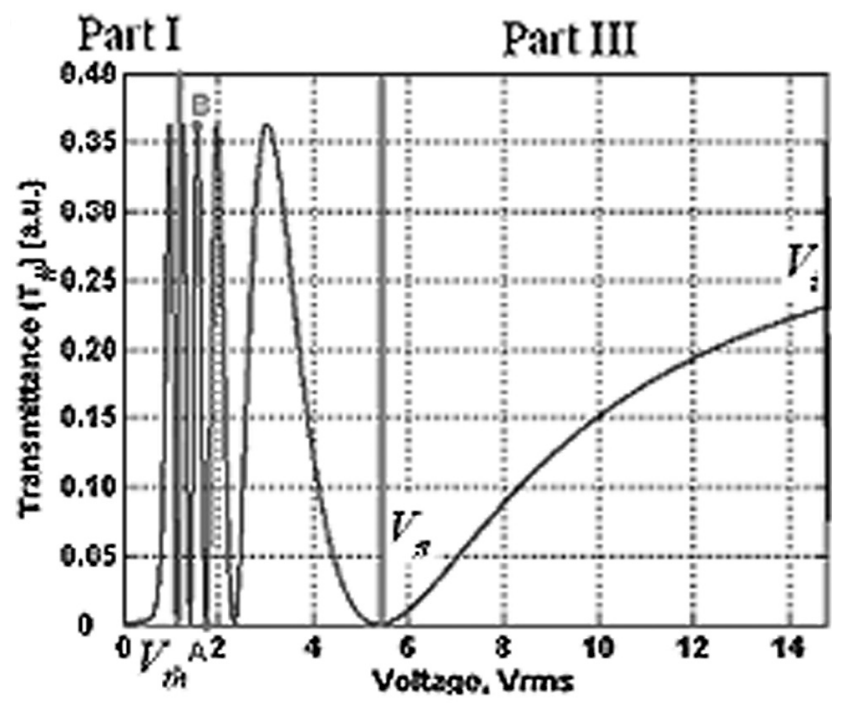

FIGURE 1 Simulated voltage-dependent transmittance curve of a $15.6-\mu \mathrm{m}$ homogeneous $\mathrm{E} 7$ cell with $3^{\circ}$ pretilt angle between paralleled polarizers at $\lambda=633 \mathrm{~nm}$ and $23^{\circ} \mathrm{C}$, where in small signal region (Part I), small angle approximation holds well; while in high signal region (Part III), the transient nematic effect is satisfied.

birefringence $(\Delta \mathrm{n})$ and low viscosity LC mixture [12-13]. The LC directors rise time $\tau_{\text {rise }}$ and decay time $\tau_{\text {decay }}$ are known to be proportional to $d^{2}$, where $d$ is the cell gap. However, the theoretical derivation of this $d^{2}$ dependence is based on the small angle approximation [14]. Thus, these equations are valid only in the $V_{t h}<V<\sqrt{2} V_{t h}$ region, marked as Part I in Figure 1, where $V$ is the applied voltage and $V_{t h}$ is the threshold voltage. Since the optical response time is linearly proportional to the LC director reorientation time [15], the corresponding optical response time is also proportional to $d^{2}$.

In the large signal regime where $V_{\pi}<V<V_{i}$, marked as Part III in Figure 1, the surface modes dominate, where $V_{\pi}$ is the voltage corresponding to last transmittance minimum and $V_{i}$ the initial voltage at the high voltage regime. As a result, both rise and decay times are fast. Moreover, the optical decay time is independent of the cell gap $d[16,17]$.

However, the optical response time in the middle voltage regime has not been studied yet. In this paper, we found that between Part I and Part III there is a region where the response time is linearly proportional to $d$. To validate this experimental observation, in Sec. II, a 
complete derivation based on small angle approximation using a parallel-aligned cell is given. Experimental methods are described in Sec. III. In Sec. IV, the validity of our analytical derivation is confirmed by experimental results and the complete physical picture of optical response time as a function of cell gap in the whole voltage regime is given.

\section{THEORY}

The voltage-dependent transmission of a $15.6 \mu \mathrm{m}$, parallel-aligned E-7 cell under parallel polarizers is shown in Figure 1. Although E-7 is chosen as an example, the results should be applicable to other LC materials. Here the maximum obtainable phase difference due to the voltage-induced molecular reorientation is $\delta=2 \pi d \Delta n / \lambda$, where $d$ is the cell gap, $\Delta n$ is the birefringence, and $\lambda$ is the wavelength. To illustrate the theory, let us divide the EO curve in Figure 1 into three parts according to the biased voltage $\mathrm{V}$.

\section{Small Signal Regime $\left(\boldsymbol{V}_{t h}<\boldsymbol{V}<\sqrt{2} \boldsymbol{V}_{t h}\right)$}

The electro-optic effect of a parallel-aligned LC layer in the small signal regime has been studied previously [14]. When $\mathrm{V}$ is not far from $\mathrm{V}_{\text {th }}\left(V_{t h}<V<\sqrt{2} V_{t h}\right)$, the small angle approximation is valid. Then the Erickson-Leslie equation under small angle approximation can be simplified to:

$$
K \frac{\partial^{2} \theta}{\partial z^{2}}+\varepsilon_{0} \varepsilon_{r} E^{2} \theta=\gamma_{1} \frac{\partial \theta}{\partial t}
$$

Under such circumstances, both rise time and decay time have simple analytical solutions, as shown in Eqs. (2) and (3), where they are both proportional to $\mathrm{d}^{2}$.

$$
\begin{gathered}
\tau_{\text {rise }}=\frac{\tau_{m}}{\left|\left(V / V_{t h}\right)^{2}-1\right|} ; \\
\tau_{\text {decay }}=\tau_{m},
\end{gathered}
$$

where

$$
\begin{gathered}
\tau_{m}=\frac{\gamma_{1} d^{2}}{K_{11} \pi^{2}} \frac{1}{(2 m+1)^{2}} \\
\tau_{0}=\frac{\gamma_{1} d^{2}}{K_{11} \pi^{2}}
\end{gathered}
$$


where $m$ stands for the number of the mode. The higher order mode (surface mode) exhibits a smaller time constant. For $\mathrm{m}=0$, the lowest spatial mode switches the slowest.

The main feature in this working scheme is that a large phase change can be achieved by a small voltage swing, which is profitable to spatial light modulators. However, this response time is relatively slow; it is proportional to $\gamma_{1} / K_{11}$ and $\mathrm{d}^{2}$, where $\gamma_{1}$ is the rotational viscosity and $K_{11}$ the splay elastic constant.

\section{Large Signal Regime $\left(\boldsymbol{V}_{\pi}<\boldsymbol{V}<\boldsymbol{V}_{\boldsymbol{i}}\right)$}

The physical mechanism of the LC device operating in this regime is the transient nematic effect. The idea of the transient nematic effect is to operate the LC device at its last transmittance cycle, from the states $V_{i}$ to $V_{\pi}$, marked as Part III in Figure 1. At this relatively high voltage regime $\left(\mathrm{V}>>\mathrm{V}_{\text {th }}\right)$, the LC directors are reoriented nearly perpendicular to the substrates except the boundary layers. So during the free relaxation process, the surface modes (the higher order modes) dominate, as shown by the solid line in Figure 2. Thus, LC device

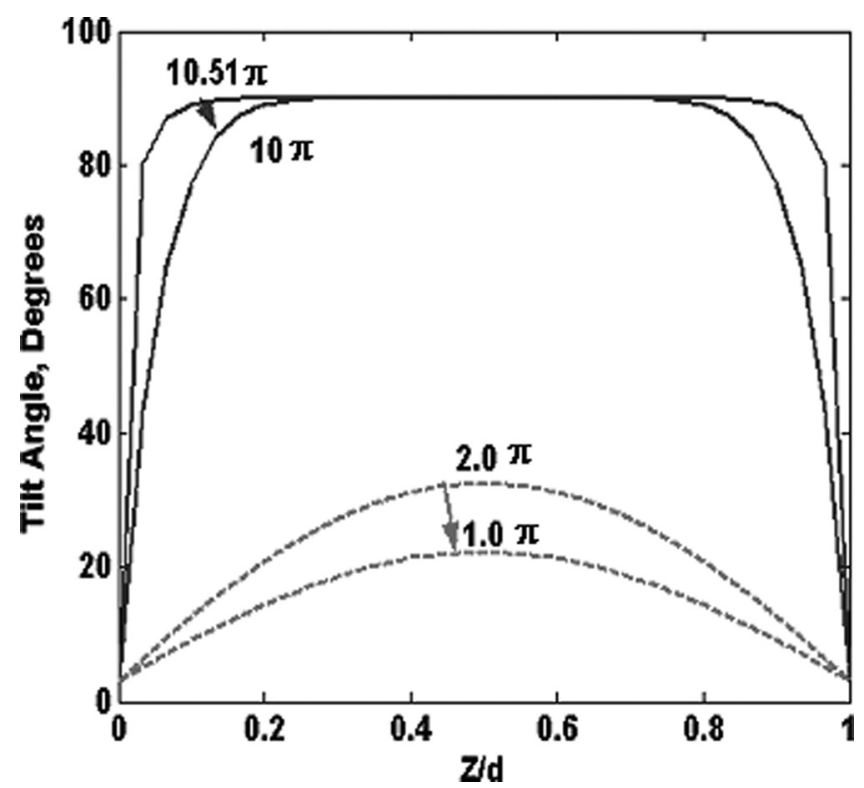

FIGURE 2 Director distribution of a $15.6-\mu \mathrm{m}$ homogeneous E7 cell between paralleled polarizers at $\lambda=633 \mathrm{~nm}$ and $23^{\circ} \mathrm{C}$. Solid line corresponds to its free decay from initial biased voltage $\mathrm{V}_{\mathrm{i}}=14.8 \mathrm{~V}_{\text {rms }}$ to its first transmittance minimum, while dotted line is its free decay from initial phase $\delta_{\mathrm{i}}=2 \pi$ to $1 \pi$. 
can reach its first minimum rapidly. Moreover, Perregaux [16] and Wu $[17,18]$ found that the optical decay time in this regime is independent of the cell gap $d$ by experimental method. Here we also verify these experimental results by numerical simulation in Sec. IV.

\section{Middle Signal Region $\left(\sqrt{2} V_{t h}<\boldsymbol{V}<V_{\pi}\right)$}

To obtain a complete physical picture of optical response time as a function of cell gap in the whole voltage regime, we study the middle signal region in this paper. Using a LC modulator to modulate light intensity, one $\pi$ phase change is usually sufficient. Thus, the objective of our study is to operate the LC device between its transmittance crest (maximum) and adjacent trough (minimum). Here we only analyze its decay process. The position of the starting point corresponds to the exactly middle part in its total phase retardation of EO curve, thus, we call it middle cycle. In Figure 1, where the total phase retardation of the LC device is $\sim 13 \pi$, the middle cycle is from point A to $\mathrm{B}$, corresponding to the LC device switching from initial phase $\delta_{\mathrm{i}} \sim 6 \pi$ (point A) to point $\mathrm{B}$, which leads to $1 \pi$ phase change.

Since the voltage-dependent intensity changes fast in the low voltage regime and saturates in the high voltage regime, indeed, the voltage $\mathrm{V}_{\mathrm{a}}$ that corresponds to the starting point of the middle cycle (point A) is not high, which is $1.8 \mathrm{~V}_{\mathrm{rms}}$ as Figure 1 shows. As the total phase retardation increases, the voltage of this point will decrease. So the small angle approximation still holds in this regime. This gives us a hint to use the small angle approximation to solve the optical response time in this region. The detailed confirming numerical results will be discussed in Sec. IV. If the voltage is removed instantaneously from point $\mathrm{A}$ at $\mathrm{t}=0$, the transient phase change can be approximated as [19]:

$$
\delta(t) \cong \delta_{i} \exp \left(-\frac{2 t}{\tau_{o}}\right)
$$

where $\delta_{i}$ is the net phase change from $\mathrm{V}=\mathrm{V}_{\mathrm{a}}$ to $\mathrm{V}=0$.

To find optical response time, we need to calculate the intensity change. The time-dependent normalized intensity change $I(t)$ of the parallel-aligned LC cell under crossed polarizers can be calculated using the following relationship

$$
I(t)=\sin ^{2}\left(\frac{\delta_{i}-\delta(t)}{2}\right) .
$$

Substituting Eq. (6) into Eq. (7), we obtain 


$$
I(t)=\sin ^{2}\left(\frac{\delta_{i}\left(1-\exp \left(-\frac{2 t}{\tau_{o}}\right)\right)}{2}\right) .
$$

Let us assume from $t_{1}$ to $t_{2}$ the transmittance decays from point $\mathrm{A}$ to point B. From Eq. (8), $I_{1}$ and $I_{2}$ have the following forms:

$$
\begin{aligned}
& I_{1}=0=\sin ^{2}\left(\frac{\delta_{o}\left(1-\exp \left(-\frac{2 t_{1}}{\tau_{o}}\right)\right)}{2}\right) \\
& I_{2}=1=\sin ^{2}\left(\frac{\delta_{i}\left(1-\exp \left(-\frac{2 t_{2}}{\tau_{o}}\right)\right)}{2}\right)
\end{aligned}
$$

Upon comparing Eq. (9) with Eq. (10), we conclude that

$$
\frac{\delta_{i}}{2} \exp \left(-2\left(t_{2}-t_{1}\right) / \tau_{0}\right)=\frac{\delta_{i}-\pi}{2} .
$$

Therefore, the solution of the optical decay time $\mathrm{T}_{\text {decay }}$ (point $\mathrm{A} \rightarrow$ point $\mathrm{B}$ ) is as follows:

$$
T_{\text {decay }}=t_{2}-t_{1}=\frac{\tau_{o}}{2} \ln \left(\frac{\delta_{i}}{\delta_{i}-\pi}\right) .
$$

Meanwhile, the initial phase $\delta_{\mathrm{i}}$ can be expressed as:

$$
\delta_{i}=\delta_{0}-\operatorname{int}\left(\delta_{0} / 2 \pi\right) \pi .
$$

Substituting Eq. (13) to Eq. (12), we can obtain the optical decay time $\mathrm{T}_{\text {decay }}$ as a function of the total phase retardation $\delta_{0}$ as:

$$
T_{\text {decay }}=\frac{\tau_{o}}{2} \ln \left(1+\frac{1}{\delta_{0} / \pi-\operatorname{int}\left(\delta_{0} / 2 \pi\right)-1}\right) .
$$

If the following assumption is made: $\delta_{0}$ is large so that $\operatorname{int}\left(\delta_{0} / 2 \pi\right)+1 \approx \delta_{0} / 2 \pi$, Eq. (14) can be greatly simplified as:

$$
T_{\text {decay }}=\frac{\gamma_{1} d}{K_{11} \pi^{2}} \frac{\lambda}{2 \Delta n}
$$

Equation (15) indicates that the optical response time $\mathrm{T}_{\text {decay }}$ in the middle cycle is linearly proportional to $d$. In the following, we will experimentally verify our theoretical prediction. 


\section{EXPERIMENT}

We prepared a set of homogeneous cells with different thickness using a commercial LC material E7. The cell gaps were determined from transmitted interference fringes through a spectrophotometer and found to be 7.8, 10.7 and $16.2 \mu \mathrm{m}$. We measured and analyzed the electro-optic characteristics of the LC cells at $\lambda=633 \mathrm{~nm}$ through a LabVIEW data acquisition system. To study the optical response times in different voltage regimes, the LC cell was initially biased at $V_{i}$. During the relaxation process, the voltage was removed at $\mathrm{t}=0$ and the optical response monitored by a photodiode detector and displayed by a digital oscilloscope.

\section{RESULTS AND DISCUSSION}

To verify our derivation in Eq. (15), we numerically solve the EricksonLeslie equation using the finite element method (FEM) [20]. To compare with experimental results, we also use E7 in our simulations.

\section{Middle Signal Regime}

The simulated transient phase change in the middle cycle of a $10-\mu \mathrm{m}$ homogeneous E7 cell with $3^{\circ}$ pretilt angle at $\mathrm{T}=23^{\circ} \mathrm{C}$ and $\lambda=633 \mathrm{~nm}$ released from $V_{i}=1.815 \mathrm{~V}_{\text {rms }}$ is depicted in Figure 3, where the solid line shows the simulated phase change, while the dash line is the fitting data in Eq. (6). The initial phase change $\delta_{0}$ is $4 \pi$. It is evident that the small angle approximation holds well in this regime, even though $\mathrm{V}_{\mathrm{i}}=1.8 \mathrm{~V}_{\text {th }}$. Thus, the assumption of our derivation is validated. Therefore, we can use the small angle approximation to solve the optical response time in this region as presented in Sec. II.

We also measured the optical decay time $t_{o p}$ in this region with these three cells. The measured results are plotted in Figure 4 (filled square). Also included in Figure 4 for comparison are the calculation results from Eq. (15) (open squares). The measured results agree well with the theoretical expectation. It is obvious that in this part the optical response time is linearly proportional to $d$.

\section{Large Signal Region}

Our study not only predict the theory of optical response time in the middle cycle, but also verify the transient nematic effect in the large signal region and small angle approximation in the small signal region. Here we only show some results in the large signal region. 


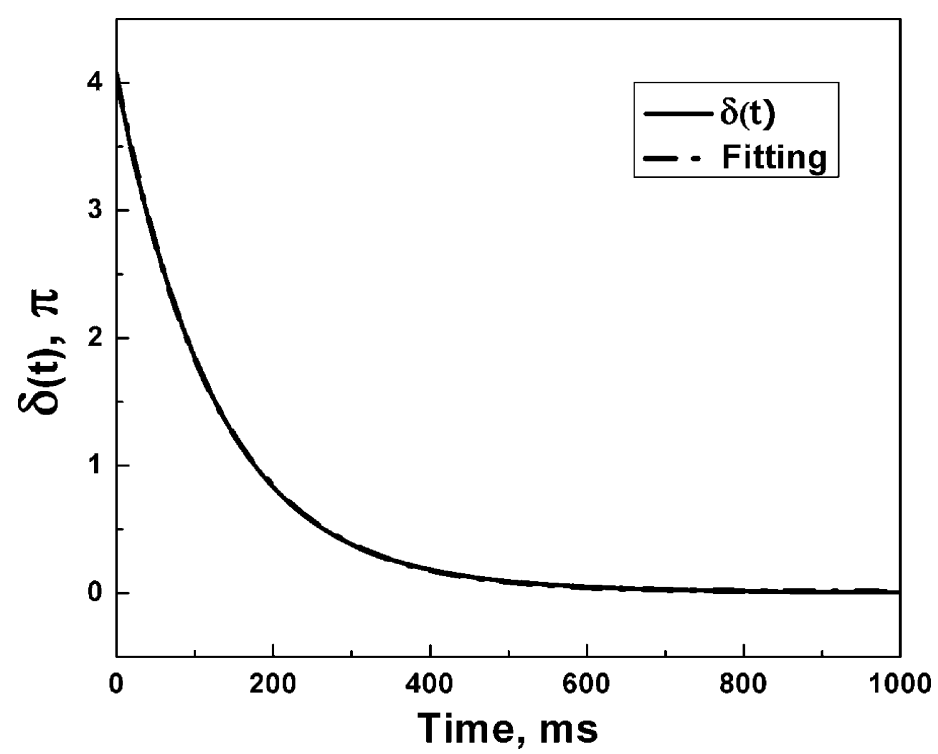

FIGURE 3 Simulated transient phase change in the middle cycle of a $10-\mu \mathrm{m}$ homogeneous E7 cell with $3^{\circ}$ pretilt angle at $\mathrm{T}=23^{\circ} \mathrm{C}$ and $\lambda=633 \mathrm{~nm}$ released from $\mathrm{V}_{\mathrm{i}}=1.815 \mathrm{~V}_{\mathrm{rms}}$. The solid line shows the simulated transient phase change, while the dash line is the fitting data using Eq. (6). Thus, the transient phase change in the middle cycle follows the small angle approximation.

In Figure 5, the above light blue line shows the experimental optical decay curve of a 10.7- $\mu \mathrm{m}$ homogeneous $\mathrm{E} 7$ cell between crossed polarizers at $\lambda=633 \mathrm{~nm}$ and $23^{\circ} \mathrm{C}$, the bottom dark blue curve is the driving square wave which is initially biased at $14.8 \mathrm{~V}_{\mathrm{rms}}$ and dropped to 0 at $\mathrm{t}=0$. The optical response time $t_{o p}$ of this cell from $\mathrm{V}_{\mathrm{i}}=14.8 \mathrm{~V}_{\mathrm{rms}}$ at the last cycle (from $t=0$ to first maximum) is $\sim 2.6 \mathrm{~ms}$. This result agrees well with the published value [19].

Figure 6 compares the results of the optical response time in the last cycle, where the red squares show the experimental optical decay times of these three cells released from $V_{i}=14.8 \mathrm{~V}_{\text {rms }}$, while the black diamonds give the simulation data. It is clear that the simulation data match the experimental results quite well. Furthermore, beyond $\mathrm{d}>10 \mu \mathrm{m}, t_{o p}$ is insensitive to $d$ as indicated in Sec. II.

Table 1 summarizes the measurement results of the optical response time $t_{o p}$ of these three cells at different voltage regimes. The optical response time in the last cycle is initially biased at $\mathrm{V}_{\mathrm{i}}=19.5 \mathrm{~V}_{\mathrm{rms}}$. Let us look at Table 1 column by column. In the last cycle, the decay time is really fast, due to the surface mode effect. 


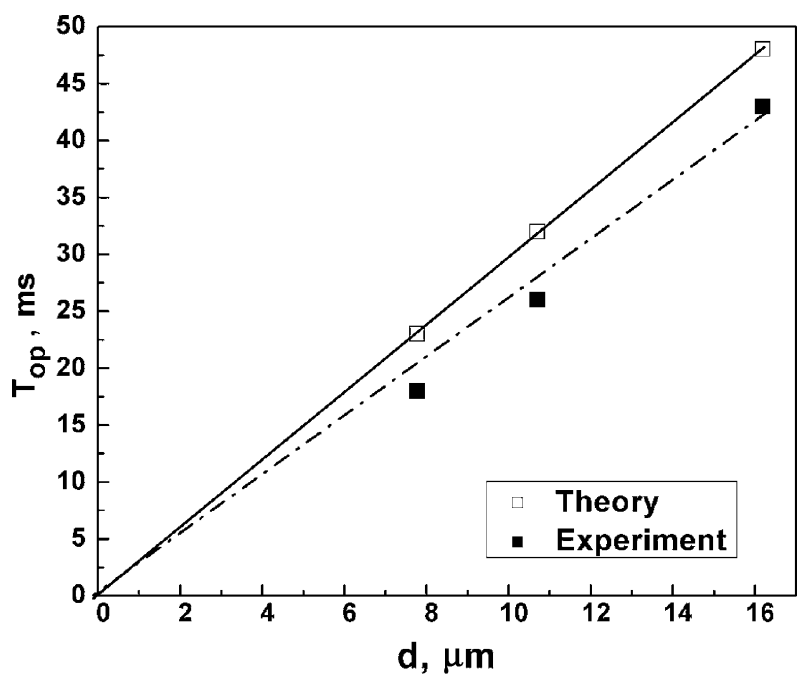

FIGURE 4 Measured and calculated optical decay times in the middle cycle as a function of the cell gap, where the filled and the empty squares show the experimental and calculation results in Eq. (15), respectively.

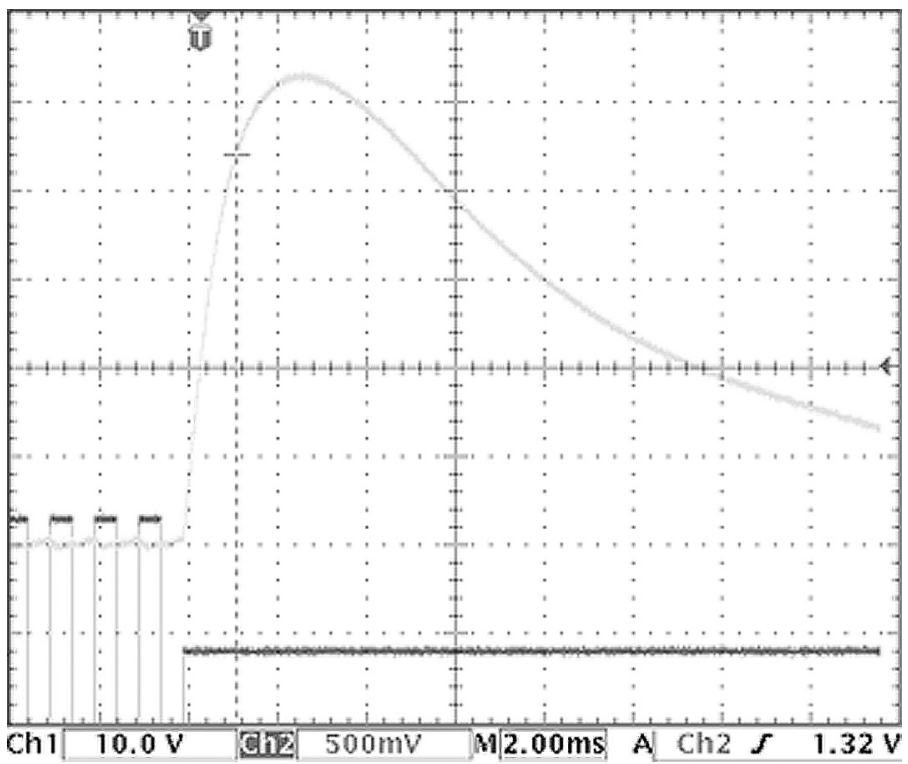

FIGURE 5 Experimental optical decay curve of a $10.7-\mu \mathrm{m}$ homogeneous E7 cell between crossed polarizers at $\mathrm{T}=23^{\circ} \mathrm{C}$ and $\lambda=633 \mathrm{~nm}$ from the initial bias voltage $\mathrm{V}_{\mathrm{i}}=14.8 \mathrm{~V}_{\mathrm{rms}}$ (light blue line). 


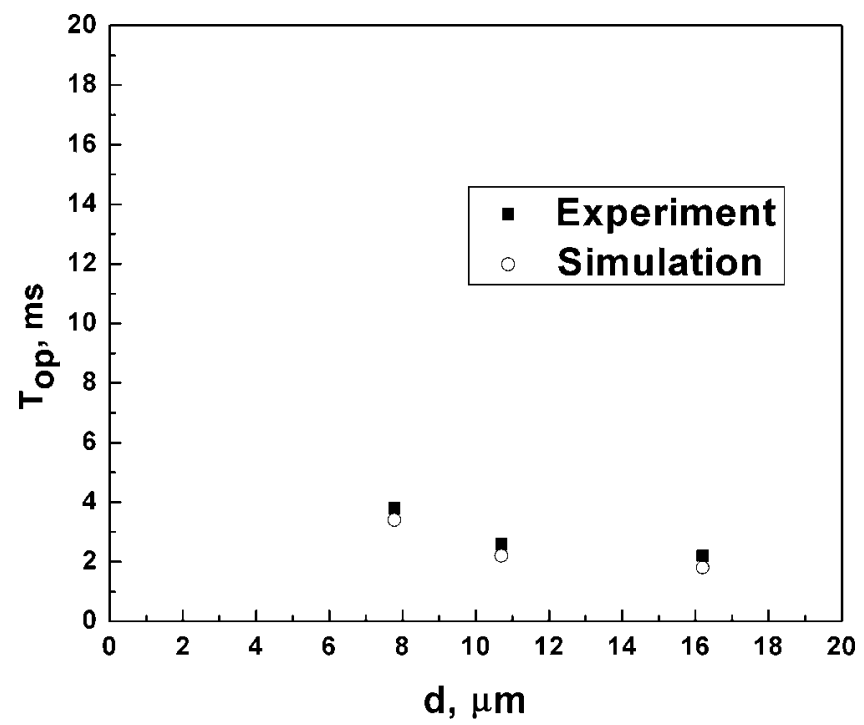

FIGURE 6 Optical response time $t_{\text {op }}$ at the last cycle as a function of the cell gap. LC used is E7 at $\mathrm{T}=23^{\circ} \mathrm{C}$ and $\lambda=633 \mathrm{~nm}$. Beyond $\mathrm{d}>10 \mu \mathrm{m}, \mathrm{t}_{\mathrm{op}}$ is insensitive to $d$ as expected in Sec. II.

TABLE I Measured Optical Response Times of Three E7 LC Cells with $\mathrm{d}=7.8,10.7$ and $16.2 \mu \mathrm{m}$ in Different Cycles. The Optical Response Time in the Last Cycle is Initially Biased at $\mathrm{V}_{\mathrm{i}}=19.5 \mathrm{~V}_{\text {rms }}$ at $\mathrm{t}=0$

\begin{tabular}{lccc}
\hline $\mathrm{d}(\mu \mathrm{m})$ & First cycle $(\mathrm{ms})$ & Middle cycle $(\mathrm{ms})$ & Last cycle $(\mathrm{ms})$ \\
\hline 16.2 & 840 & 43 & 2.2 \\
10.7 & 250 & 26 & 2.4 \\
7.8 & 80 & 18 & 2.5 \\
\hline
\end{tabular}

Moreover, the optical response time is independent of the cell gap. In the middle cycle, the measured response time, as the filled square plotted in Figure 4, is linearly proportional to the cell gap. In the first cycle, because of the smaller elastic torque, the optical response time is relatively slow. Furthermore, the measured optical response time is proportional to $d^{2}$ as predicted by the theory.

\section{CONCLUSION}

We have analyzed theoretically and confirmed experimentally that the optical response time in the middle phase cycle of the EO curve is 
linearly proportional to $d$. The analytical solution of the optical response time is derived based on the small angle approximation. The confirming experimental and the simulation results agree well with the theoretical expectation. Therefore, in the whole voltage regime, the physical picture of optical response time as a function of cell gap is completed. This analysis is useful for understanding the grayscale switching behaviors of the LC phase modulators.

\section{REFERENCES}

[1] McManamon, P. F., Dorschner, T. A., Corkum, D. L., Friedman, L., Hobbs, D. S., Holz, M., Liberman, S., Nguyen, H. Q., Resler, D. P., Sharp, R. C., \& Watson, E. A. (1996). Proc. of the IEEE, 84, 268.

[2] Sato, S. (1979). J. Appl. Phys., 18, 1679.

[3] Love, G. D., Major, J. V., \& Purvis, A. (1994). Opt. Lett., 19, 1170.

[4] Presnyako, V. V., Asatryan, K. E., \& Galstian, T. V. (2002). Opt. Express, 10, 865.

[5] Ren, H. \& Wu, S. T. (2002). Appl. Phys. Lett., 81, 3537.

[6] Ren, H. \& Wu, S. T. (2003). Appl. Phys. Lett., 82, 22.

[7] Chou, J., Han, Y., \& Jalali, B. (2003). IEEE Photon. Technol. Lett., 15, 1041.

[8] Sneh, A. \& Johnson, K. M. (1996). J. Light. Technol., 14, 1067.

[9] Kibe, S., Hattori, N., Ushioda, M., Yamamoto, H., \& Matsui, S. (2003). J. SID, $11,449$.

[10] Geelhaar, T., Tarumi, K., \& Hirschmann, H. (1996). Soc. Inf. Display Tech. Digest, $27,167$.

[11] Goto, Y., Ogawa, T., Sawada, S., \& Sugimori, S. (1991). Mol. Cryst. Liq. Cryst., 209, 1.

[12] Fan, Y. H., Lin, Y. H., Ren, H., Gauza, S., \& Wu, S. T. (2004). Appl. Phys. Lett., 84, 1233.

[13] Lu, Y., Du, F., Lin, Y. H., \& Wu, S. T. (2004). Opt. Express, 12, 1221.

[14] Jakeman, E. \& Raynes, E. P. (1972). Phys. Lett. A, 39, 69.

[15] Wang, H., Wu, T. X., Zhu, X., \& Wu, S. T. (2004). J. Appl. Phys., 95, 5502.

[16] Perregaux, A. E. (1986). U. S. Patent 4,595,259.

[17] Wu, S. T. \& Wu, C. S. (1988). Appl. Phys. Lett., 53, 1794.

[18] Wu, S. T. \& Wu, C. S. (1989). J. Appl. Phys., 65, 527.

[19] Wu, S. T. \& Wu, C. S. (1990). Phys. Rev. A, 42, 2219.

[20] Akin, J. E. (1994). Finite Elements for Analysis and Design, Academic Press: San Diego, CA. 